\title{
. \\ iNAPO - lon Conducting Nanopores in Polymer Foils Chemically Modified for Biomolecular Sensing
}

\author{
Wolfgang Ensinger ${ }^{1}$, Gerhard Thiel ${ }^{2}$, Ivana Duznovic ${ }^{1}$, Saima Nasir ${ }^{1}$, Mubarak Ali ${ }^{1}$ \\ Technische Universität Darmstadt \\ ${ }^{1}$ Department of Materials Science, Materials Analysis, Darmstadt, Germany \\ ensinger@ma.tu-darmstadt.de,duznovic@ma.tu-darmstadt.de, nasir@ma.tu-darmstadt.de,m.ali@gsi.de \\ ${ }^{2}$ Department of Biology, Membrane Biophysics, Darmstadt, Germany \\ thiel@bio.tu-darmstadt.de
}

\begin{abstract}
In the present biomimetic approach, ion-conducting nanopores are fabricated in polymer foils and are chemically modified so that they can be used as the core of a biomolecular sensing system. The basic principles of fabrication and working mechanism of these nanosensors is described. Polyimide foils are irradiated at the heavy ion accelerator at GSI Helmholtz-Center in Darmstadt with a single ion of a heavy element such as gold. The ion is at such a high speed that it penetrates through the membrane in a straight line, forming a so-called latent ion damage track. This track is converted into a single conical nanopore via asymmetric chemical etching, with its smaller aperture being in the range of a few nanometers. At the surface of the nanopore a biorecognition unit (ligand) is immobilized which specifically fit to another molecule (receptor) to be analysed. Only with these analyte molecules recognition unit do react in a keylock principle. For sensing, the polymer foil with the nanopore is placed into an electrochemical cell with two compartments, with the foil acting as separation membrane. Each compartment contains an electrode dipped in electrolyte (potassium chloride) solution. When a potential is applied, an electric current flows through the nanopores. If analyte molecules (receptor) are present, they react with the immobilized ligand at the nanopore walls via specific ligand-receptor interaction, thus changing the transmembrane electrolyte current. Thus, the set-up works as a highly sensitive and specific nanosensor. Fabrication and working principle of this type of nanosensor is described. As an example, results on quantitative sensing of a protein are shown. As an outlook for development and application of this sensing principle, the $\boldsymbol{i N A P O}$-project will be presented, run by a group of materials scientists, biologists, chemists, physicist and electrical engineers with the aim of creating new types of sensors based on bioinspired ion conducting nanopores.
\end{abstract}

Keywords: nanopores, biochemical sensor, current-potential measurements, ion track etching, nanosensor

\section{Introduction}

All membranes of living organisms contain proteins for the selective and regulated transport of either ions, water or small molecules [1]. From a mechanistic point of view ion channels (ion conducting nanopores) are the most interesting among the various forms of membrane transport proteins. They form water filled transmembrane protein tunnels, which are able to transport ions with a velocity, which approaches free diffusion of ions in water. In spite of this high transport velocity channels are still highly selective; $\mathrm{K}^{+}$channels for example are able to discriminate between cations and transport the larger $\mathrm{K}^{+}$up to 1000 times better than the smaller $\mathrm{Na}^{+}$. The operation of ion channels is characterized by a stochastic fluctuation between conductive "open" states and non-conductive "closed" states. This process of gating regulates the current flow across membranes and is a crucial parameter for the regulation of channel activity in the physiological context. Many physical factors such as voltage or mechanical forces but also chemical factors like ligands or signaling molecules can directly or indirectly modulate the activity of channels. Inspired by these sophisticated biological systems, there are currently many attempts to engineer sensors on the basis of these protein nanopores. One example is the stochastic sensing of analytes by channel proteins, which were engineered in such a way that they alter their gating in the presence of molecules of interest [2].

A major drawback of biological nanochannels/pores is that they developed throughout evolution in living cells as membrane-embedded proteins. This makes them rather fragile and renders them essentially not suitable for most technical applications. In order to overcome these restrictions, various routes have been tried for fabricating nanopores in solid-state 
materials. The goal of these endeavors is to create solid-state pores, which mimic functions of biological nanopores and combine in this way the robustness of the solid state material with the sensoric sensitivity and selectivity of biological material [3]. The materials which are commonly used range from silicon, silicon nitride, glass, and alumina to polymers. Techniques for preparing the nanochannels include laser ablation, highly localized electron beam heating, and focused ions for ion beam sculpting. Another approach, employed in the present studies, is the so-called ion track-etching technique. Here, polymer foils are through-irradiated with a beam of highly energetic ions in a particle accelerator. The ion damage track is preferentially chemically etchable into a nanopore. This can be done asymmetrically so that conical nanopores are obtained [4]. Such nanopores can be used as center parts of electrochemical sensing devices.

The goal of the $i N A P O$ project is the combination of biological protein-based and polymer-based nanopores and their implementation in a micro-nano-device in order to create a new type of micro-tool for sensing and analytical applications, e.g. for environmental or medical applications, such as water analysis and diagnostics. In the following, the fundamental of fabrication of the nanopores and their use as biomolecular sensor is described.

\section{Experimental Details}

Polyimide foils (PI, Kapton $50 \mathrm{HN}$, DuPont) of $12 \mu \mathrm{m}$ thickness were irradiated at the linear accelerator UNILAC (GSI Helmholtz-Center of Heavy Ion Research, Darmstadt, Germany) with a single gold ion with a kinetic energy of 2.2 $\mathrm{GeV}$. With such an immense energy, the ion crosses the polymer foil completely along a straight line. It transfers a large amount of energy to the electrons of the material, typically several $1000 \mathrm{eV}$ per $\mathrm{nm}$ track length. As a consequence of this extremely large electronic excitation, chemical bonds are broken and small volatile fragments are cut out of the polymer leaving back a zone of reduced density and lower chemical stability.

In order to fabricate an asymmetric nanopore, the PI foil is clamped between two compartments of an electrochemical cell, with one part filled with an etching solution (sodium hypochlorite), the other one with a so-called stopping solution, which neutralizes the etchant, here potassium iodide. The etchant removes the polymer in the ion damage track much faster than the undamaged material thus creating a conical pore. The large aperture of the conical pore is typically in the region of several hundred $\mathrm{nm}$, up to $\mu \mathrm{m}$, the smaller one a few $\mathrm{nm}$, i.e. a nanoscale diameter region is obtained.

In order to check the conical shape of the nanopores, a replica technique is used. For this purpose, the polymer foil is coated on one side with a thin film of gold by sputter deposition. Then, metal is galvanically deposited inside the nanopore. Here, copper sulphate was used to fill the nanopore with copper. After deposition, the polymer was dissolved and the copper "needle" was inspected under the scanning electron microscope.

Since the small aperture of the nanopores is in the nanometric region and cannot be resolved accurately by the electron microscope, its dimension is measured via determining its electrolyte conductance, based on Ohm's law. Here, an electrolyte current flowing through the nanopores is measured. The ionic current is directly proportional to the nanopore dimensions. The set-up is shown in Fig. 2.
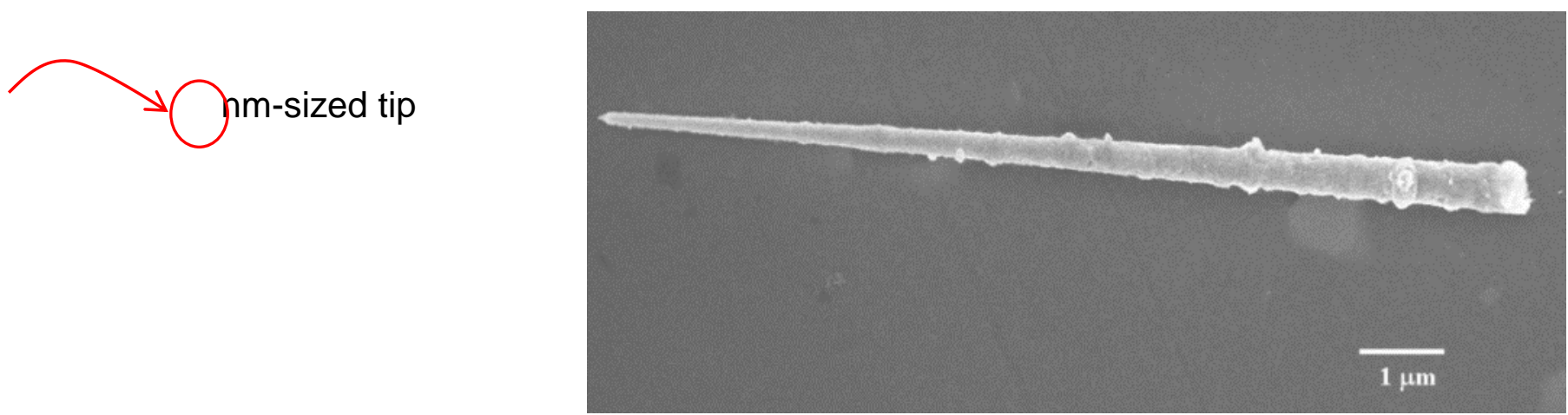

Fig. 1: Scannning electron micrograph of a copper replica of the conical nanopore. The nanopore was galvanically filled with copper and the polymer foil acting as template was removed.

When the inner nanopore walls need to be chemically functionalized, an appropriate coupling chemistry is required. Covalent coupling via EDC/PFP ( $N$-(3-dimethyl-aminopropyl)- $N$ '-ethylcarbodiimide hydrochloride and penta-fluorophenol) turned out to be a reliable process [5]. First, the terminal carboxylic acid group on the nanopore surface was converted into 
pentafluorophenyl ester with an ethanolic solution of EDC and PFP. The reactive-PFP-ester was then submitted to a reaction with 4-aminophenol $\alpha$-D-mannopyranoside (APMP), exposing mannose as a biorecognition element at the nanopore wall.

The nanopore works as an electrochemical sensor where an ionic electrolyte current $I$ through the nanopore is measured as a function of an applied voltage $V$. The set-up is shown in Fig. 2. For the voltammetric $I / V$ measurements, the foil with the functionalized nanopore was mounted in between the two compartments of an electrochemical cell made of Teflon. An aqueous $0.1 \mathrm{M} \mathrm{KCl}$ solution was used as an electrolyte. Each cell compartment contained a standard reference electrode, connected to a voltage source and a sensitive current meter (picoammeter 6487, Keithley Instruments, Cleveland, $\mathrm{OH})$. Upon applying a voltage ramp from $-2 \mathrm{~V}$ to $+2 \mathrm{~V}$ between the electrodes in steps of $0.1 \mathrm{~V}$, the current flowing through the nanopore was measured. Solutions of Concanavalin A, a model analyte, with different concentrations (from 10 nanomolar to 1500 nanomolar) were used. Concanavalin A is a carbohydrate-binding protein (legume lectine) composed of 235 amino acids.
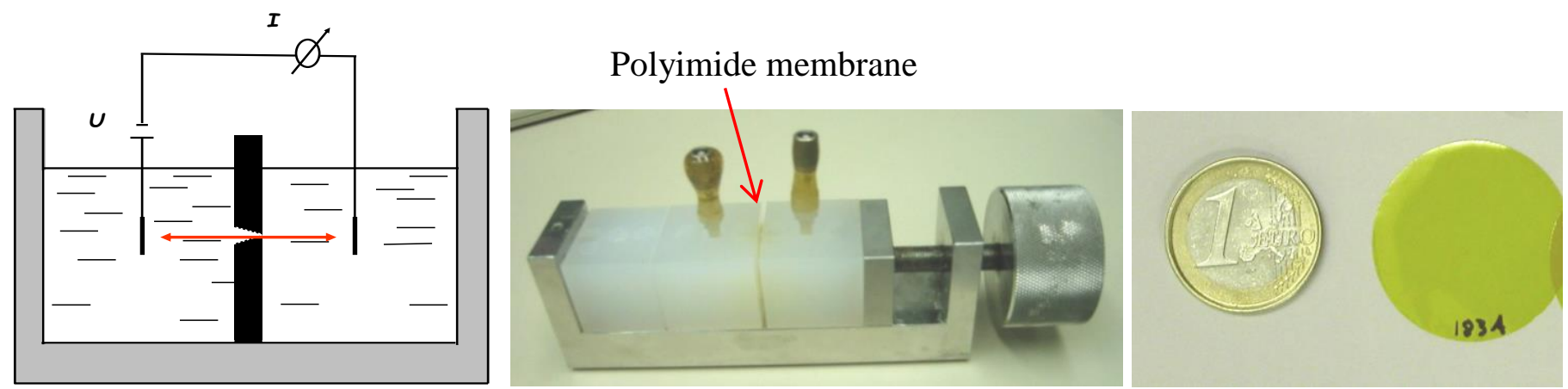

Fig. 2: Schematic presentation of electrochemical set-up consisting of a two-compartment cell with two electrodes. The polymer foil with the conical nanopore is shown in black. The transmembrane ionic current path of the electrolyte ions $\left(\mathrm{K}^{+}, \mathrm{Cl}^{-}\right)$is shown in red. The pressure-sealed Teflon cell with the polymer foil in the center and the electrode contacts at each side is depicted in the middle. The polyimide foil with a nanopore in its center is shown at the right hand side, together with a $1 €$ coin.

\section{Results and Discussion}

The working principle of the nanopore sensor is based upon the ligand-receptor interactions at nanoscale dimension, i.e. its nanometric diameter. Since its length is not of relevance, it belongs to the 1D-nanostructures. The ability of the electrolyte ions to pass through the nanopore driven by the outer potential is influenced by two major effects, one being trivially the nanopore diameter, the other one being the surface charge inside the nanopore. In the first case, the ionic current through the nanopore will be changed if large (bio)molecules are anchored at the nanopore wall via a specific reaction of reactive groups at the nanopore surface. In the second case, the ionic transport will be influenced if the analyte molecules (receptor) to be detected react with counter parts (ligand) immobilized at the nanopore wall, and upon this reaction the surface charge/diameter of the nanopore wall is changed. This directly becomes visible in the current conducted by the current.

For a specific reaction of the analyte molecule with the nanopore surface, the saccharide mannose molecules were immobilized by EDC/PFP coupling chemistry at the nanopore wall with its terminal carboxyl (-COOH) groups. The analyte (receptor) to be measured was Concanavalin A (ConA) protein, a lectin, which possesses specific binding sites for saccharides, i.e. it bioconjugates with high selectivity to mannose [6]. The coupling of ConA to the mannopyranoside (APMP) leads to a partial occlusion of the nanopore and, as a consequence, reduced ionic currents through the nanopore, as schematically shown in Fig. 3 [7]. 

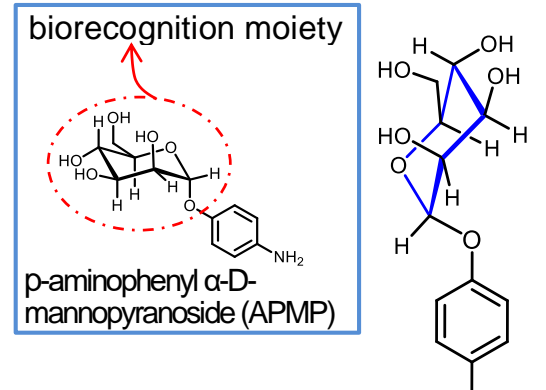<smiles>CC(C)(C)C(=O)O</smiles>

(a)

(b)

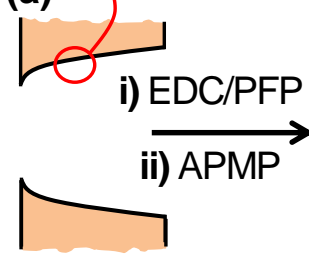

bound mannoside-ConA

bioconjugate

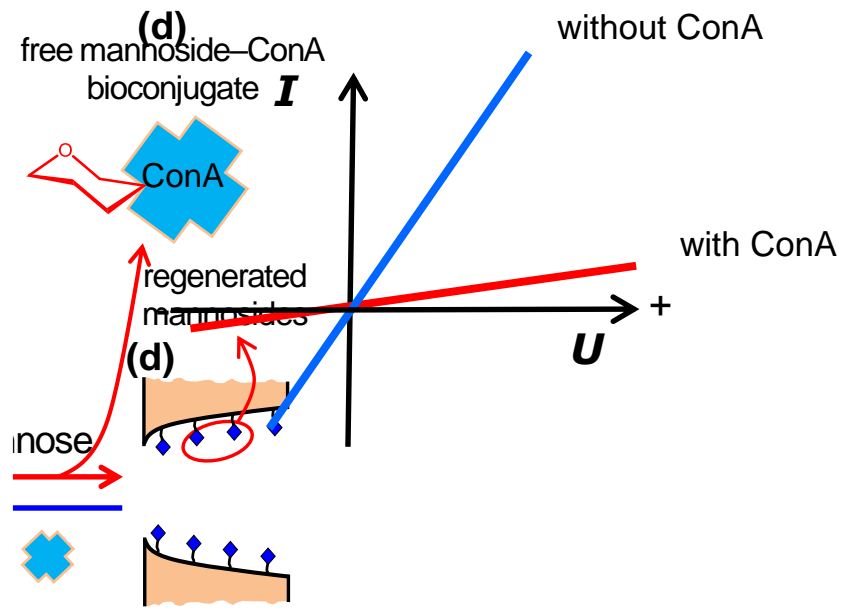

Fig. 3: a) The nanopore with its terminal carboxyl groups is schematically shown in cross-section. b) Mannose is immobilized at the wall via EDC/PFP coupling chemistry by the amino group of APMP. c) Concanavalin A specifically couples to mannose, leading to a reduction in nanopore diameter [7], d) schematic presentation of nanopore current-voltage curves with and without ConA.

Fig. 4 shows the nanopore current for different concentrations of ConA after the bioconjugation process inside the nanopores, Also, the resulting nanopore conductance is depicted. It turns out that the nanopore-based sensor is able to quantify ConA over a wide range of concentrations from as little as $10 \mathrm{nM}$ (or probably less) to more than $1 \mathrm{mM}$.
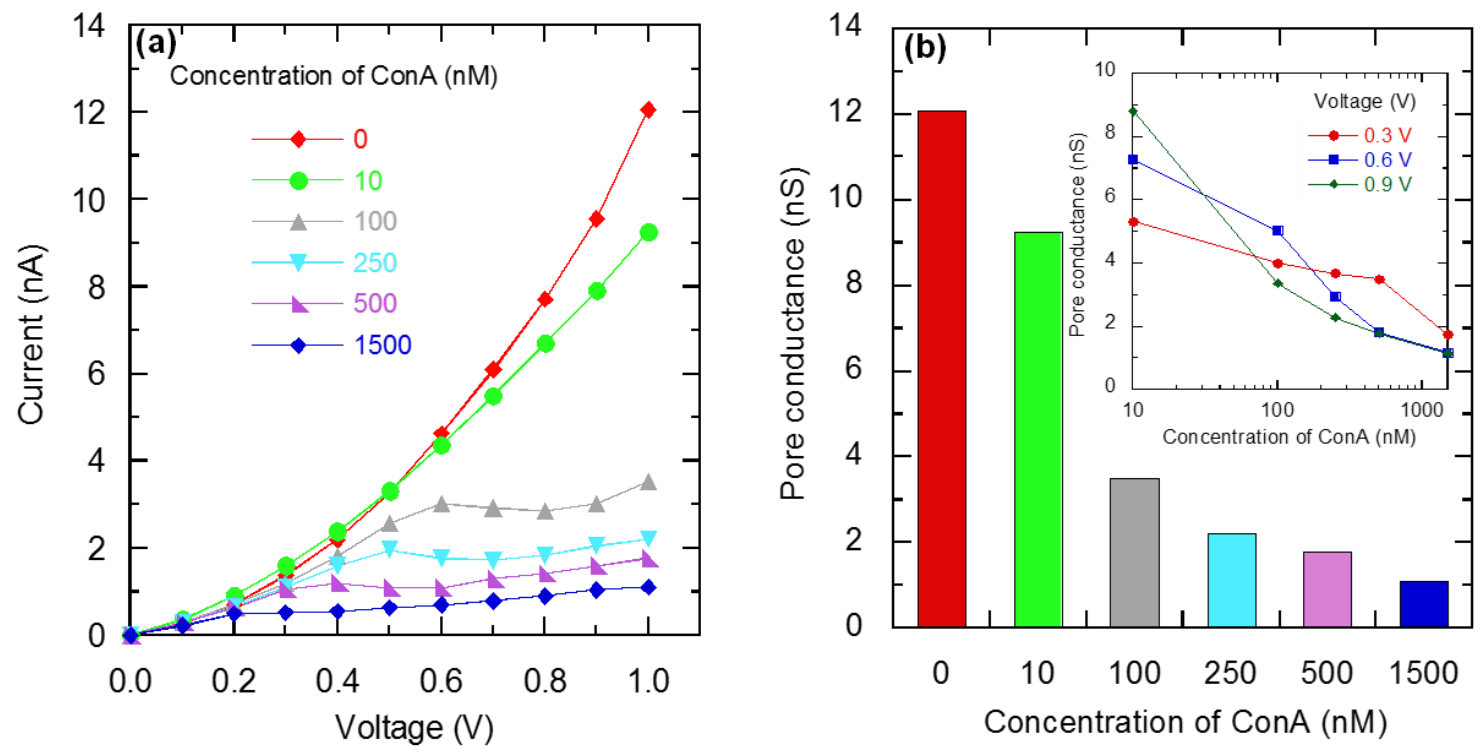

Fig. 4: a) Current-voltage curves of the ionic transmembrane current for different concentrations of ConA. b) The pore conductance derived from the response current, shown for different ConA concentrations and voltages, is a direct function of the concentration [7]. The error is typically in the range of a few $\%$. 
The presence of other proteins in similar or even higher quantities, such as Lysozyme or Bovine Serum Albumin, does not affect the measurement, since they do not react with mannose i.e. the sensor is specific to ConA.

Repetition of the measurements and evaluation gave an error in quantification of a few percent only.

Other types of interaction have been reported elsewhere, e.g. with boronic acid [8], aptamers [9], or based on metal ion - protein interaction [10], and with enzymatic reactions, e.g. for hydrogen peroxide sensing [11, 12].

\section{Conclusion}

It has been demonstrated that chemically modified single nanopores in polymer foils in combination with electrochemical measurement of the nanopore ion conductance work very well as biomolecular sensors. Their sensitivity is essentially given by the nanoscale dimensions of the system. The small aperture of the conical nanopores is of the same dimension as that of various biomolecules. The molecular selectivity depends on the specific "key-lock" reaction between the analyte molecule and its counterpart immobilized on the nanopores walls. The concept of the functionalized ion conducting nanopores can be applied to a large number of biorecognition couples.

Within the project $i N A P O$, the potential of this technique will be further explored and exploited. Protein-based nanopores with even better selectivity will be embedded into polymer foils. Eventually, the nanopore foils will be incorporated in electronic micro sensing devices with low volume and energy consumption, thus creating a new type of biomolecular sensor. Applications aim towards e.g. environmental analysis and medical diagnostics.

\section{Acknowledgements}

This work has been supported in the frame of the LOEWE project $i N A P O$ by the Hessen State Ministry of Higher Education, Research and the Arts.

\section{References}

[1] B. Hille, Ionic channels of excitable membranes. Sunderland, MA: Sinauer Associates Inc., 2001.

[2] H. Bayley and P. S. Cremer, "Stochastic sensors inspired by biology," Nature, vol. 413, pp. 226, 2001.

[3] A. J. Storm. J. H Chen, X. S. Ling, H. W. Zandbergen, and C. Dekker, "Fabrication of solid-state nanopores with singlenanometre precision," Nat. Mater., vol. 2, pp. 537, 2003.

[4] P. Y. Apel, Y. E. Korchev, Z. Siwy, R. Spohr, and M. Yoshida, "Diode-like single-ion track membrane prepared by electro-stopping," Nucl. Instrum. Methods Phys. Res., B, vol. 184, pp. 337, 2001.

[5] M. Ali, V. Bayer, B. Schiedt, R. Neumann, and W. Ensinger, "Fabrication and functionalization of single asymmetric nanochannels for electrostatic/hydrophobic association of protein molecules," Nanotechnology, vol. 19, pp. 485711, 2008.

[6] Z. Derewenda, J. Yariv, J. R. Helliwell, A. J. Kalb, E. J. Doson, M. Z. Papiz, T. Wan, and J. Campbell, "The structure of the saccharide-binding site of concanavalin A," EMBO J., vol. 8, pp. 2189, 1989.

[7] M. Ali, S. Nasir, P. Ramirez, J. Cervera, S. Mafe, and W. Ensinger, "Carbohydrate-mediated biomolecular recognition and gating synthetic nanochannels," J. Phys. Chem., vol. C117, pp. 18234, 2013.

[8] Q. H. Nguyen, A. Ali, R. Neumann, and W. Ensinger, "Saccharide/glycoprotein recognition inside synthetic ion channels modified with boronic acid," Sens. Actuators B., vol. 162, pp. 216, 2012.

[9] M. Ali, S. Nasir, and W. Ensinger, "Bioconjugation-induced ionic current rectification in aptamer-modified single cylindrical nanopores," Chem. Comm., vol. 51, pp. 3454, 2015.

[10] M. Ali, S. Nasir, H. Q. Nguyen, J. K. Sahoo, M. N. Tahir, W. Tremel, and W. Ensinger, "Metal Ion Affinity-based Biomolecular Recognition and Conjugation inside Synthetic Polymer Nanopores Modified with Iron-Terpyridine Complexes," J. Am. Chem. Soc., vol. 133, pp. 17307, 2011.

[11] M. Ali, M. N. Tahir, Z. Siwy, R. Neumann, W. Tremel, and W. Ensinger, "Hydrogen Peroxide Sensing with Horseradish Peroxidase-Modified Polymer Single Conical Nanochannels," Anal. Chem., vol. 83, pp. 1673, 2011.

[12] M. Ali, I. Ahmed, S. Nasir, P. Ramirez, C. M. Niemeyer, S. Mafe, and W. Ensinger, "Ionic Transport through Chemically Functionalized Hydrogen Peroxide-Sensitive Asymmetric Nanopores," ACS Appl. Mater. Interfaces, vol. 7, pp. 19541, 2015. 